\title{
MODELAGEM BIOECONÔMICA DA TRANSFERÊNCIA DE EMBRIÕES EM BOVINOS
}

\author{
Renato Travassos Beltrame, ${ }^{1}$ Luis Gustavo Barioni, ${ }^{2}$ Celia Raquel Quirino ${ }^{3}$ e Ozanival Dario Dantas ${ }^{2}$ \\ 1. Mestre em Produção Animal, doutorando em Ciência Animal, LRMGA, CCTA, UENF. \\ E-mail: rtbeltrame@terra.com.br \\ 2. Pesquisador da EMBRAPA \\ 3. Universidade Estadual do Norte Fluminense
}

RESUMO

\begin{abstract}
O objetivo deste trabalho foi desenvolver um modelo matemático orientado a eventos de simulação, para auxiliar tomadas de decisão relativas à transferência de embriões em bovinos, considerando-se as dinâmicas de dois componentes da transferência de embriões: receptoras e embriões. Na simulação, não se avaliaram respostas individuais de doadoras a coletas consecutivas e eventos correspondentes na transferência de embriões. Simulou-se o mesmo protocolo para superovulação a todas as doadoras. Receptoras foram sincronizadas simulando-se o uso de prostaglandina. O número de embriões viáveis produzido por doadora e sua variabilidade
\end{abstract}

tiveram como base um processo aleatório de simulação de Monte Carlo, que pressupôs uma distribuição exponencial negativa de densidade de probabilidade. Custos e receitas foram inseridos no modelo por meio de um cenário-base para calcular indicadores econômicos de rentabilidade. A análise sugeriu a impraticabilidade da atividade, se realizada diante do cenário proposto (VPL - R\$: $57.596,69)$. A partir do cenário proposto, o custo médio estimado foi de $\mathrm{R} \$ 1.178,19$, e de $\mathrm{R} \$ 980,03$, para se obter uma prenhez a partir de uma situação otimizada, sugerida pelo modelo $(5 / 100 ; 5 / 190)$.

PALAVRAS-CHAVES: Otimização, receptoras, simulação, transferência de embriões, viabilidade econômica.

ABSTRACT

\section{BIOECONOMIC MODEL IN BOVINE EMBRYO TRANSFER}

A simulation model related to embryo transfer programs in bovine was carried out through a mathematical model directed to events, considering the dynamic of two resources: recipients and embryos. Individual answers of donors to consecutive collections and corresponding events in embryo transfer were not evaluated. The same protocol for superovulation was simulated for all the donor collections, using similar doses of hormones and drugs for all the animals. Recipients were synchronized using prostaglandin. Meantime, the number of viable embryos produced by donor and its variability were based at Monte Carlo random simulation process, which presupposed a negative exponential distribution of density of probability. Costs were inserted in the model through a basis scenery to calculate economic indicators of profitability (cost per pregnancy, Net Present Value and Internal Rate of Return). The analysis suggested the impracticality of the activity if it is carried out in the proposed scenery NPV (US\$ - 25,042.04). An estimate average cost of US\$ 512.25 and of US\$ 426.01 was obtained by the proposed scenery to get a pregnancy at an optimized situation, $(5 / 100 ; 5 / 190)$.

KEY WORDS: Optimization, recipients, simulation, embryo transfer, economic viability.

\section{INTRODUÇÃO}

Programas de transferência de embriões em bovinos (TE) dependem da disponibilidade de receptoras que permitam o desenvolvimento de uma gestação a termo (REICHENBACH et al., 2002). A compra e a manutenção de receptoras correspondem a uma considerável proporção dos custos da TE (FERNANDES, 
1999; BELTRAME et al., 2007), sendo de extrema importância na determinação do número de produtos que podem ser gerados. Ainda, a identificação do número ótimo de receptoras é de grande valor econômico (BARIONI et al., 2007; BELTRAME et al., 2007).

Entretanto, a grande variabilidade na produção de embriões pelas doadoras (SLENNINGL et al., 1989; GALLI et al., 2003) e a necessidade de compra e sincronização de receptoras antes que se conheça o número de embriões coletados dificultam a decisão acerca do número de animais que devem ser destinados à sincronização.

Usualmente, o número de receptoras a serem alocados para um programa de TE é calculado com base no número médio de embriões coletados por doadora (BÓ et al., 2004). Embora grande parte das estimativas em trabalhos envolvendo aspectos reprodutivos seja trabalhada de forma determinista (FRIGGENS et al, 2005), alguns trabalhos têm sugerido o uso de números aleatórios e geração estocástica de variáveis (OSTERGAARD et al., 2005).

Na tentativa de resolver problemas e projetar antecipadamente resultados de atividades que envolvam elevado risco, alguns modelos matemáticos têm sido desenvolvidos, permitindo auxílio à tomada de decisões nas diversas esferas em sistemas de produção na espécie bovina (TEDESCHI et al., 2005). Alguns deles têm estudado aspectos reprodutivos das fêmeas bovinas (SLENNINGL et al., 1989; OSTERGAARD et al., 2005; BARIONI 2007; BELTRAME et al., 2007).

O objetivo deste trabalho foi desenvolver um modelo matemático para auxiliar na tomada de decisões acerca da transferência de embriões em bovinos, determinando a viabilidade econômica e o número ótimo de receptoras por doadora em cenários simulados.

\section{MATERIAL E MÉTODOS}

Neste estudo foi desenvolvido um modelo de simulação para auxiliar tomadas de decisão a respeito de aspectos econômicos relativos à TE. Para simular um programa de TE, utilizou-se um modelo matemático orientado a eventos (PERIN FILHO, 1995), considerando a dinâmica de dois importantes componentes: receptoras e embriões.

Na dinâmica de receptoras, a simulação se inicia pela compra de receptoras. Após a aquisição, um período de tempo é considerado para que ocorra uma adaptação dos animais. Assim, nesse processo, computaram-se as ações referentes à aplicação de vacinas, de vermífugos, à avaliação reprodutiva e à obtenção de escore de condição corporal (ECC) positivo. $\mathrm{O}$ início da TE ocorreu simultaneamente à entrada de receptoras no grupo de sincronização. Para a realização de uma TE, pressupõe-se uma sincronia entre receptoras e doadoras. Dessa forma, um protocolo de sincronização de receptoras é definido considerando-se a aplicação de fármacos. Nesse momento, as receptoras são conduzidas a um processo de sincronização correspondente ao período necessário para que os fármacos administrados promovam seus efeitos e, com isso, sejam produzidos os parâmetros de avaliação da resposta. Mediante a utilização de um protocolo de sincronização de receptoras para transferência de embriões por tempo fixo (TETF), condiciona-se a totalidade dos animais respondendo ao evento estro, sendo realizada a palpação retal para identificação do corpo lúteo (CL), caracterizando a ovulação. As receptoras que não apresentam CLretornam ao grupo de sincronização. Aquelas detectadas com a estrutura luteínica são destinadas ao grupo de receptoras aptas.

Para protocolos que utilizam a aplicação intervalada de prostaglandina $\left(\mathrm{PGF}_{2 \mathrm{alfa}}\right)$, após a aplicação de fármacos, as receptoras passam à sincronização. Após a detecção de estro, as receptoras que não foram detectadas retornam ao grupo de sincronização. Caso contrário, se destinam ao evento de palpação retal. Nesse evento, as receptoras que não apresentam CL retornam ao grupo de sincronização. Aquelas que apresentam CL são destinadas ao grupo de receptoras aptas.

É importante ressaltar que o processo de sincronização não incorpora a dinâmica temporal do ciclo estral. O tempo até a entrada no grupo de receptoras aptas é considerado conforme a escolha do protocolo de sincronização. Entretanto, os parâmetros de resposta não estão condicionados ao tempo.

O evento de inovulação somente ocorrerá caso existam embriões viáveis (coletados a fresco ou congelados) e receptoras aptas.

As receptoras inovuladas são direcionadas ao processo aguardando diagnóstico de gestação. Sequencialmente, um evento de diagnóstico de gestação pode desencadear o aumento do estoque de receptoras prenhes confirmadas ou o acréscimo de receptoras prontas para sincronizar. 
Como o modelo pressupõe o uso de uma mesma receptora por apenas dois ciclos, ao final do eventodiagnóstico, algumas receptoras podem ser destinadas ao grupo de descarte, ao término dos ciclos.

Após a colheita, os embriões obtidos podem, diante da disponibilidade no grupo de receptoras aptas, ser inovulados a fresco ou, diante da escassez destas, serem congelados.

O modelo modifica o estoque de embriões congelados nas seguintes situações: quando o número de receptoras aptas ocorre em excesso; quando a produção de embriões excede o número de receptoras aptas.

Os eventos de congelamento e inovulações com embriões descongelados são controlados pelo estoque de embriões congelados e pelo grupo de receptoras aptas. Ainda, esses eventos estão condicionados ao evento inovulação. Quando se excede o número de embriões produzidos em relação ao número de receptoras aptas, o evento de congelamento é realizado. No entanto, pode haver situações em que existam escassez de embriões e, consequentemente, sobra de receptoras aptas. Nesse caso, um descongelamento de embriões em estoque é realizado, e um evento de inovulação é lançado à simulação.

\section{Modelagem matemática}

Como a dinâmica de doadoras extrapola os objetivos deste trabalho, não foram avaliadas respostas individuais de doadoras a coletas consecutivas e eventos correspondentes a sua utilização na TE.

As equações matemáticas de um modelo de TE quantificam as diversas etapas da biotécnica. Assim, como exposto por BARIONI et al. (2007), se não houvesse variação nos coeficientes técnicos considerados, particularmente no número de embriões viáveis obtidos por coleta, as estimativas deterministas seriam precisas na determinação de resultados. Entretanto, o número de embriões varia consideravelmente entre doadoras e para uma mesma doadora em diferentes coletas. Alia-se a isso a necessidade de receptoras serem adquiridas e sincronizadas antes que se tenha conhecimento sobre o número de embriões viáveis a ser obtido a partir das doadoras superovuladas. Assim, mesmo utilizando-se o número ideal de receptoras, haverá, por vezes, número excessivo de embriões coletados e, por outras, número insuficiente para as receptoras aptas à inovulação.
No caso de haver excesso de embriões, é possível congelá-los para futuras inovulações. A possibilidade de congelamento implica um estoque de embriões que varia ao longo do tempo, dependendo do número de embriões produzidos e do número de receptoras aptas em cada coleta.

Partindo do descrito por BARIONI et al. (2007) e visando contemplar o estoque de embriões, utilizouse um modelo matemático dinâmico no qual o tempo foi discretizado em intervalos mensais. Entretanto, a coleta de embriões, o número de embriões viáveis gerados por doadora e sua variabilidade tiveram por base um processo aleatório de simulação de Monte Carlo (PERIN FILHO, 1995), com frequência esperada que pressupõe uma distribuição exponencial negativa de densidade de probabilidade, conforme sugerido por BELTRAME (2006).

As possibilidades de congelamento, de descongelamento e de movimentação do estoque de embriões foram matematicamente similares às descritas por BARIONI et al. (2007), sendo demonstradas nas seguintes equações:

$$
\mathrm{G}_{\mathrm{i}--}=\mathrm{I}_{\mathrm{f}} \alpha_{\mathrm{gf}}+\mathrm{I}_{\mathrm{c}} \alpha_{\mathrm{gc}}
$$

em que $\mathrm{G}_{\mathrm{i}}$ é o número de gestações obtidas por coleta, $\mathrm{I}_{\mathrm{f}}$ é o número de inovulações com embriões frescos e $\mathrm{I}_{\mathrm{c}}$ é o número de inovulações com embriões congelados. Os parâmetros $\alpha_{\mathrm{gf}} e \alpha_{\mathrm{gc}}$ são respectivamente as taxas de concepção de embriões inovulados a fresco e inovulados após descongelamento.

O número de inovulações a fresco pode ser limitado pelo número de embriões viáveis coletados $\left(\mathrm{E}_{\mathrm{i}}\right)$ ou pelo número de receptoras aptas $\left(\mathrm{A}_{\mathrm{i}}\right)$. Portanto, pode ser calculado como o mínimo entre os valores dessas variáveis:

$$
I_{f}=\operatorname{Min}\left(E_{i}, A_{i}\right.
$$

Inovulações a partir de embriões congelados ocorrem somente quando o número de receptoras aptas é maior que o número de embriões viáveis coletados, isto é, quanto $\mathrm{A}_{\mathrm{i}}-\mathrm{E}_{\mathrm{i}}>0$. $\mathrm{O}$ número de receptoras que pode ser inovulado é o mínimo entre o número de receptoras aptas não inovuladas a fresco $\left(\mathrm{A}_{\mathrm{i}}-\mathrm{E}_{\mathrm{i}}\right)$, e o número de embriões congelados em estoque $\left(\mathrm{C}_{\mathrm{i}}\right)$. Essas premissas são expressas algebricamente pela seguinte equação:

$$
\mathrm{I}_{\mathrm{c}}=-\operatorname{Min}\left(\Delta \mathrm{C}_{\mathrm{i}}, 0\right) \text {, }
$$

em que $\Delta \mathrm{C}_{\mathrm{i}}$ é a variação no estoque de embriões congelados, $\Delta \mathrm{C}_{\mathrm{i}}>0$ indica que houve congelamento, $\Delta \mathrm{C}_{\mathrm{i}}<0$ indica que houve inovulação. Assim, uma 
variação negativa $\left(\Delta \mathrm{C}_{\mathrm{i}}<0\right)$ indica que $-\Delta \mathrm{C}_{\mathrm{i}}$ embriões foram descongelados e inovulados $\left(\mathrm{Ic}=-\Delta \mathrm{C}_{\mathrm{i}}\right)$. Uma variação positiva indica que houve congelamento $\mathrm{e}$ não inovulações e, nesse caso, $\operatorname{Min}\left(\Delta \mathrm{C}_{\mathrm{i}}, 0\right)=0 . \Delta \mathrm{C}_{\mathrm{i}}$ é calculado de acordo com esta equação:

$$
\Delta \mathrm{C}_{\mathrm{i}}=\operatorname{Max}\left(\mathrm{E}_{\mathrm{i}}-\mathrm{I}_{\mathrm{i}}-\mathrm{C}_{\mathrm{i}}\right) \text {, }
$$

em que $\quad C_{i}=\sum_{j-1}^{i-1} \Delta C_{i} ; C_{0}=0$

Como o objetivo do modelo não pressupõe a comparação da eficiência de doadoras, um mesmo protocolo de superovulação foi utilizado para todas as coletas, sendo consideradas doses de hormônios e fármacos similares para todas as doadoras. Esse protocolo teve como base a utilização de dipositivo de progesterona $\left(\mathrm{CIDR}^{\circledR}\right.$ ), estrógeno (Estrogin $\left.{ }^{\circledR}\right)$, FSH (Foll$\left.\operatorname{tropin}^{\circledR}\right)$, PGF2 $\alpha\left(\right.$ Ciosin $\left.^{\circledR}\right)$ e GnRH (Gestran Plus $\left.{ }^{\circledR}\right)$. Consideraram-se duas inseminações e recuperação embrionária no $16^{\circ}$ dia após início do tratamento.

A sincronização de receptoras foi projetada de forma a possibilitar a comparação dos índices de eficiência e variáveis econômicas. Projetou-se um protocolo dependente de observação do estro (aplicação de prostaglandina) seguindo o descrito por SPELL et al. (2001).

As estimativas de custo inseridas no modelo tiveram como intuito permitir o cálculo de indicadores econômicos de rentabilidade valor presente líquido (VPL) e taxa interna de retorno (TIR) (PENEDO, 2005) e a determinação do número ótimo de receptoras (R/D), diante do protocolo de sincronização de receptoras utilizado. Essas estimativas tiveram como base valores de mercado pesquisados em outubro de 2005 , e paridade de câmbio de U\$S 1,00 para $\mathrm{R} \$ 2,30$. A taxa de desconto utilizada foi de $6 \%$ ao ano.

Para a inserção dos custos no modelo, foram sugeridas cinco categorias, que englobaram as possibilidades de localização das receptoras no modelo, conforme ilustra a Tabela 1 .

$\mathrm{Na}$ Tabela 1, o cálculo do custo do item "adaptação" inclui manutenção nutricional dos animais por um mês (custo de oportunidade sobre o arrendamento de pastagem) mais vermífugos, vacinas, honorários veterinários, exames. Os custos das receptoras nas categorias "prontas para sincronizar" e "aguardando diagnóstico" são relativos exclusivamente à manutenção mensal nutricional da receptora.
TABELA 1. Estimativa de custo em reais correspondente à localização das receptoras no modelo

\begin{tabular}{lc}
\hline \multicolumn{1}{c}{ Item } & Custo mensal \\
\hline Adaptação & $\mathrm{R} \$ 50,84$ \\
Receptoras prontas para sincronizar & $\mathrm{R} \$ 26,33$ \\
Receptoras aptas & $\mathrm{R} \$ 41,13$ \\
Aguardando diagnóstico de gestação & $\mathrm{R} \$ 26,33$ \\
Diagnóstico de gestação & $\mathrm{R} \$ 28,04$ \\
\hline
\end{tabular}

O custo de "receptoras aptas" engloba o somatório dos custos mensais de manutenção, o custo do protocolo de sincronização utilizado e, caso haja inovulação, engloba também os custos de antibioticoterapia com dihidroestreptomicina.

A estimativa de custo referente à categoria "diagnóstico de gestação" foi obtida pelo somatório da manutenção mensal à avaliação reprodutiva.

Nas estimativas, consideraram-se os custos de aquisição de receptoras $1 / 2$ sangue Bos Taurus x Bos Indicus, adquiridas a um peso médio de 360 quilos. No descarte das receptoras, assumiu-se um peso de 420 quilos. O preço de venda adotado partiu do valor médio para o Estado de São Paulo obtido pela vaca gorda nos últimos nove anos (ANUALPEC, 2005). Nesse caso, os preços de aquisição e de descarte da receptora considerados foram, respectivamente, de $\mathrm{R} \$$ 500,00 e de $\mathrm{R} \$ 583,00$.

Como o objetivo do trabalho visa identificar se os ganhos propiciados pela tecnologia superam os custos adicionais que ela acarreta, é importante destacar que custos inerentes à atividade pecuária, tais como instalações, manutenções e custos indiretos, não foram aqui considerados.

Para efeito de desenvolvimento do modelo e no intuito de aproximar as estimativas de custo à realidade, assumiu-se que todas as doadoras foram coletadas consecutivamente por três vezes considerando-se, então, a partir daí, uma gestação a termo. É importante ressaltar que não se considerou "covariância" entre coletas para uma mesma doadora e, portanto, a geração de embriões respeitou o descrito por BELTRAME (2006).

Para doadoras, o modelo oferece a possibilidade de inclusão ou não dos custos de aquisição de valor residual e de manutenção das doadoras que efetivamente não estão no programa. Estes cálculos são demonstrados pelas seguintes equações: 


$$
\begin{aligned}
& \mathrm{PC}=(\mathrm{R}-1) * \mathrm{I}+60 \\
& \mathrm{PT}=\mathrm{I}_{\mathrm{c}}^{*} \mathrm{C}+120 \\
& \mathrm{DP}=\left(\mathrm{C} * \mathrm{D} * \mathrm{I}_{\mathrm{c}}\right) / \mathrm{R} \\
& \mathrm{DM}=(\mathrm{DP} / 4)^{*} \mathrm{I}_{\mathrm{c}}
\end{aligned}
$$

em que PC é o período de coleta mínimo por doadora em dias; PT é o período total do programa em dias; DP é o somatório do número de doadoras do programa; DM é o número de doadoras em manutenção; Ic é o intervalo entre coletas e tem como valor de referência trinta dias, devendo, nesse caso, ser utilizado na fórmula o valor " 1 ". No caso de valores superiores ou inferiores a trinta dias, seu valor deve ser, respectivamente, reduzido ou ampliado proporcionalmente à referência "1"; D é o número de doadoras em coleta; I é o período de descanso entre coletas; R é o número de coletas sucessivas para uma doadora e $\mathrm{C}$ é o número de coletas do programa.

Um cenário-base, originado a partir de índices tradicionais de desempenho encontrados na bibliografia (SPELL et al., 2001; BÓ et al., 2004; NASSER et al., 2004), foi proposto e aplicado ao modelo. Nesse cenário, foram definidas quantas doadoras seriam trabalhadas em cada coleta, o período de simulação, o intervalo entre coletas, o tempo de adaptação, o intervalo protocolo - TE, o tempo para diagnóstico de gestação, o protocolo de sincronização da receptora à ciclicidade do rebanho, a taxa de sincronização das receptoras, a taxa de aptidão e taxas de gestação a fresco e de descongelamento (Tabela 2).

TABELA 2. Índices e períodos estabelecidos no cenário-base para doadoras em coleta

\begin{tabular}{lr}
\hline Item & Padrão \\
\hline Número de doadoras por coleta & 5 \\
Período de simulação (anos) & 10 \\
Intervalo entre coletas (dias) & 30 \\
Adaptação (dias) & 50 \\
Intervalo protocolo/cio (dias) & 14 \\
Intervalo cio/TE (dias) & 7 \\
Diagnóstico de gestação (dias) & 60 \\
Protocolo de sincronização da receptora & 70 \\
Ciclicidade do rebanho (\%) & 70 \\
Taxa de sincronização das receptoras (\%) & 60 \\
Taxa de aptidão (\%) & 50 \\
Taxa de gestação a fresco (\%) & 35 \\
Taxa de gestação por descongelamento (\%)
\end{tabular}

Fonte: Modificado de SPELL et al. (2001).
Os custos foram informados ao modelo conforme método apresentado na Tabela 1. Além desses custos, inseriram-se os campos para o custo de aquisição da doadora, valor residual da doadora, valor de descarte da receptora, custo de manutenção da doadora, custo do protocolo de superovulação e valor de venda da prenhez, como demonstrado na Tabela 3.

TABELA 3. Valores considerados no cenário-base em uma fazenda

\begin{tabular}{lr}
\hline Item & Base (R\$) \\
\hline Custo de aquisição da doadora & $15.000,00$ \\
Valor residual da doadora & $15.000,00$ \\
Valor de descarte da receptora & 583,00 \\
Custo do protocolo de adaptação & 54,31 \\
Custo de manutenção da receptora & 26,33 \\
Custo de sincronização da receptora & 12,00 \\
Custo de manutenção da doadora & 82,66 \\
Custo do protocolo de superovulação & 252,20 \\
Honorários veterinários por doadora em coleta & 500,00 \\
Valor de venda da prenhez & $1.500,00$ \\
Taxa de desconto (\%) ano & 6,0 \\
\hline
\end{tabular}

O cenário proposto visou contemplar a atividade em uma propriedade, em que índices e custos tradicionais da TE fossem introduzidos como dados de entrada. Assim, possibilitou-se a determinação de índices de viabilidade dessa atividade na situação proposta. Nesse caso, custos de doadoras, receptoras e índices de eficiência foram completamente inseridos no modelo, possibilitando a determinação e a especulação do VPL e da TIR.

No cenário-base, o modelo foi utilizado para determinar e projetar um fluxo de caixa da atividade, estimando número de gestações produzidas, receitas provenientes do descarte de receptoras, da venda de gestações e índices econômicos ao final do período.

Para se observar o comportamento desse cenário durante um período maior de atividade e seu comportamento otimizado (BM), foi realizada uma análise de sensibilidade em função do tempo e do número de receptoras por doadoras utilizadas.

O custo de manutenção de doadoras foi somente contabilizado para os animais que estavam em coleta, permanecendo todo o restante constante. Assim, 
buscou-se identificar, na situação proposta, o custo de produção de uma prenhez e índices de rentabilidade. Nessa situação, para o cálculo de viabilidade da atividade, sugeriu-se um valor de venda da prenhez de $\mathrm{R} \$ 1.500,00$.

As simulações foram executadas 5.000 vezes em cada caso, estabelecendo médias do número de prenhezes e índice de eficiência do cenário elaborado. Os resultados de número de prenhezes foram comparados pelo Teste de Tukey, sendo extrapolados para a comparação monetária realizada.

\section{RESULTADOS E DISCUSSÃO}

Os resultados apresentados a seguir foram obtidos com o simulador implementado e destacam as principais variáveis físicas e econômicas para a realização da atividade em uma fazenda.

O modelo implementado foi capaz de gerar resultados a partir das informações estabelecidas para entrada. Ainda, otimizou o uso dos animais no cenário criado (base), visto sugerir dados de entrada diferentes para a variável número de receptoras, proporcionando, nesse caso, valores inferiores de custo para produção das prenhezes (cenário $\mathrm{BM})$.

Pela análise do VPL (Tabelas 4, 5 e 6), no cenário proposto a atividade é inviável. Nesse caso, como VPLé simplesmente a diferença entre o valor presente do projeto e o custo do projeto na data atual, este custa mais do que vale, ou seja, se for implementado trará prejuízo (ABREU FILHO, 2003).

Embora o cenário criado apresente comportamento pessimista, demonstrado pelo VPL negativo, e taxa interna de retorno baixa, tal fato pode ser decorrente dos índices de eficiência aplicados e do número de receptoras utilizado. Os resultados da otimização do cenário proposto sugerem que 190 receptoras devam ser utilizadas, ao contrário das 100 impostas inicialmente. Dessa maneira, considerando-se os índices reprodutivos do cenário-base e o tempo simulado, a viabilidade de execução da atividade e de retorno do capital empregado é demonstrada na Tabela 6 . Entretanto, maiores estudos devem ser realizados no intuito de comprovar valores positivos para VPL, caso se execute a atividade por um horizonte de tempo diferente do proposto na simulação e diante de cenários diferentes.
A partir do cenário proposto, chegou-se a uma estimativa de custo médio de R $\$ 1.178,19$ por prenhez confirmada. FERNANDES et al. (2006) demonstram uma análise ex-post com resultados contrastantes aos demonstrados neste trabalho. Embora o valor médio de cada produto nascido viável não tenha ultrapassado $\mathrm{R} \$ 442,74$, no trabalho apresentado pelos citados autores, o tipo de análise, a metodologia de cálculo e taxas de gestações superiores alcançadas podem ter sido responsáveis pelas diferenças encontradas.

Comparou-se a eficiência de protocolos de sincronização de receptoras utilizando-se como critério o custo da prenhez. BELTRAME et al. (2007), simulando dois anos de coletas, mantiveram o número de receptoras fixo e utilizaram distribuição normal com média seis e desvio-padrão seis para simular a geração do número de embriões viáveis por coleta e identificar mínimo custo por prenhez e razão ideal de uso de receptoras e doadoras. As estimativas de custo variaram de $\mathrm{R} \$ 553,40$ quando o número ideal de receptoras por doadora foi utilizado, a $\mathrm{R} \$ 1.150,00$, quando se empregaram outras razões de receptoras por doadoras (BELTRAME et al., 2007).

BARIONI et al. (2007) compararam modelos deterministas e estocásticos em programas de transferência de embriões em bovinos submetidos a análises com e sem congelamento de embriões. Embora nas análises deterministas não tenham ocorrido diferenças em termos de estimativas de custo da prenhez, o congelamento foi fundamental para reduzir o custo da prenhez nas estimativas estocásticas. As estimativas de custo variaram de $\mathrm{R} \$$ 434,12 a R\$1.490,92, conforme a variabilidade da produção de embriões e do custo de receptoras analisadas.

TABELA 4. Saídas da simulação dos cenários-base e BM

\begin{tabular}{|c|c|c|}
\hline Cenários & Base & $\mathrm{BM}$ \\
\hline Número de doadoras & 5 & 5 \\
\hline Número de receptoras & 100 & 190 \\
\hline Receptoras $v s$ doadora & 20 & 38 \\
\hline Número de gestações & $893^{\mathrm{a}}$ & $1681^{\mathrm{b}}$ \\
\hline Custo da prenhez & $\mathrm{R} \$ 1.178,19$ & $\mathrm{R} \$ 980,03$ \\
\hline VPL & $R \$-56.595,39$ & $\begin{array}{r}\mathrm{R} \$ \\
235.479,82\end{array}$ \\
\hline TIR & $0,16 \%$ & $22,71 \%$ \\
\hline
\end{tabular}

${ }^{(1)}$ Médias seguidas por letras iguais não diferem entre si pelo teste de Tukey, a $5 \%$ de probabilidade. 
TABELA 5. Fluxo de caixa em reais $(\mathrm{R} \$)$ previsto para o cenário-base

\begin{tabular}{|c|c|c|c|c|c|}
\hline Ano & 0 & 1 & 2 & 3 & 4 \\
\hline \multicolumn{6}{|l|}{ Despesas } \\
\hline Compra de receptoras & $94.000,00$ & $59.500,00$ & $60.000,00$ & $59.000,00$ & $60.000,00$ \\
\hline Adaptação & $10.210,28$ & $6.462,89$ & $6.517,20$ & $6.408,58$ & $6.517,20$ \\
\hline Compra de doadoras & $75.000,00$ & 0 & 0 & 0 & 0 \\
\hline Manutenção mensal das receptoras & $32.175,26$ & $28.462,73$ & $28.436,40$ & $28.489,06$ & $28.436,40$ \\
\hline Manutenção mensal das doadoras & $4.546,30$ & $4.959,60$ & $4.959,43$ & $4.959,43$ & $4.959,43$ \\
\hline Sincronização & $4.872,00$ & $5.064,00$ & $5.088,00$ & $5.112,00$ & $5.076,00$ \\
\hline Honorários veterinários & $25.000,00$ & $30.000,00$ & $30.000,00$ & $30.000,00$ & $30.000,00$ \\
\hline Superovulação & $12.610,00$ & $15.132,00$ & $15.132,00$ & $15.132,00$ & $15.132,00$ \\
\hline \multicolumn{6}{|l|}{ Receitas } \\
\hline Valor das gestações & $99.000,00$ & $136.500,00$ & $135.000,00$ & $135.000,00$ & $133.500,00$ \\
\hline Descarte de receptoras & $6.996,00$ & $16.324,00$ & $17.490,00$ & $16.324,00$ & $18.073,00$ \\
\hline \multicolumn{6}{|l|}{ Valor residual das doadoras } \\
\hline Total & $-152.417,84$ & $3.242,78$ & $2.356,97$ & $2.222,93$ & $1.451,97$ \\
\hline Ano & 5 & 6 & 7 & 8 & 9 \\
\hline \multicolumn{6}{|l|}{ Despesas } \\
\hline Compra de receptoras & $59.000,00$ & $60.500,00$ & $59.000,00$ & $59.000,00$ & $69.000,00$ \\
\hline Adaptação & $6.408,58$ & $6.571,51$ & $6.408,58$ & $6.408,58$ & $7.494,78$ \\
\hline Compra de doadoras & - & - & - & - & - \\
\hline Manutenção mensal das receptoras & $28.489,06$ & $28.410,07$ & $28.489,06$ & $28.489,06$ & $35.597,46$ \\
\hline Manutenção mensal das doadoras & $4.959,43$ & $4.959,43$ & $4.959,43$ & $4.959,43$ & $6.199,50$ \\
\hline Sincronização & $5.112,00$ & $5.028,00$ & $5.064,00$ & $5.088,00$ & $5.916,00$ \\
\hline Honorários veterinários & $30.000,00$ & $30.000,00$ & $30.000,00$ & $30.000,00$ & $32.500,00$ \\
\hline Superovulação & $15.132,00$ & $15.132,00$ & $15.132,00$ & $15.132,00$ & $16.393,00$ \\
\hline \multicolumn{6}{|l|}{ Receitas } \\
\hline Valor das gestações & $138.000,00$ & $132.000,00$ & $132.000,00$ & $129.000,00$ & $165.000,00$ \\
\hline Descarte de receptoras & $15.158,00$ & $19.239,00$ & $17.490,00$ & $18.656,00$ & $74.624,00$ \\
\hline Valor residual das roadoras & & & & & $75.000,00$ \\
\hline Total & $4.056,93$ & 637,99 & 436,93 & $-1.421,07$ & $141.523,26$ \\
\hline VPL & $-57.596,69$ & & & & \\
\hline TIR & $0,16 \%$ & & & & \\
\hline
\end{tabular}


TABELA 6. Fluxo de caixa em reais $(\mathrm{R} \$)$ previsto para o cenário BM

\begin{tabular}{|c|c|c|c|c|c|}
\hline Ano & 0 & 1 & 2 & 3 & 4 \\
\hline \multicolumn{6}{|l|}{ Despesas } \\
\hline Compra de receptoras & $184.500,00$ & $112.500,00$ & $112.500,00$ & $112.000,00$ & $114.000,00$ \\
\hline Adaptação & $20.040,39$ & $12.219,75$ & $12.219,75$ & $12.165,44$ & $12.382,68$ \\
\hline Compra de doadoras & $75.000,00$ & & & & \\
\hline Manutenção mensal de receptoras & $61.559,54$ & $54.108,15$ & $54.108,15$ & $54.134,48$ & $54.029,16$ \\
\hline Manutenção mensal de doadoras & $4.546,30$ & $4.959,60$ & $4.959,60$ & $4.959,60$ & $4.959,60$ \\
\hline Sincronização & $7.800,00$ & $9.636,00$ & $9.696,00$ & $9.708,00$ & $9.624,00$ \\
\hline Honorários veterinários & $25.000,00$ & $30.000,00$ & $30.000,00$ & $30.000,00$ & $30.000,00$ \\
\hline Superovulação & $12.610,00$ & $15.132,00$ & $15.132,00$ & $15.132,00$ & $15.132,00$ \\
\hline \multicolumn{6}{|l|}{ Receitas } \\
\hline Valor das gestações & $175.500,00$ & $253.500,00$ & $250.500,00$ & $247.500,00$ & $256.500,00$ \\
\hline Descarte de receptoras & $8.745,00$ & $32.648,00$ & $33.814,00$ & $34.397,00$ & $33.231,00$ \\
\hline \multicolumn{6}{|l|}{ Valor residual das doadoras } \\
\hline Total & $(206.811,23)$ & $47.592,50$ & $45.698,50$ & $43.797,48$ & $49.603,56$ \\
\hline Ano & 5 & 6 & 7 & 8 & 9 \\
\hline \multicolumn{6}{|l|}{ Despesas } \\
\hline Compra de receptoras & $113.000,00$ & $111.500,00$ & $113.500,00$ & $113.000,00$ & $132.000,00$ \\
\hline Adaptação & $12.274,06$ & $12.111,13$ & $12.328,37$ & $12.274,06$ & $14.337,84$ \\
\hline \multicolumn{6}{|l|}{ Compra de doadoras } \\
\hline Manutenção mensal de receptoras & $54.081,82$ & $54.160,81$ & $54.055,49$ & $54.081,82$ & $67.562,78$ \\
\hline Manutenção mensal de doadoras & $4.959,60$ & $4.959,60$ & $4.959,60$ & $4.959,60$ & $6.199,50$ \\
\hline Sincronização & $9.648,00$ & $9.732,00$ & $9.648,00$ & $9.672,00$ & $11.304,00$ \\
\hline Honorários veterinários & $30.000,00$ & $30.000,00$ & $30.000,00$ & $30.000,00$ & $32.500,00$ \\
\hline Superovulação & $15.132,00$ & $15.132,00$ & $15.132,00$ & $15.132,00$ & $16.393,00$ \\
\hline \multicolumn{6}{|l|}{ Receitas } \\
\hline Valor das gestações & $252.000,00$ & $250.500,00$ & $256.500,00$ & $255.000,00$ & $322.500,00$ \\
\hline Descarte de receptoras & $33.814,00$ & $32.648,00$ & $32.648,00$ & $32.648,00$ & $139.337,00$ \\
\hline Valor residual das doadoras & & & & & $75.000,00$ \\
\hline Total & $4.056,93$ & 637,99 & 436,93 & $-1.421,07$ & $141.523,26$ \\
\hline VPL & $237.075,82$ & & & & \\
\hline TIR & $22,71 \%$ & & & & \\
\hline
\end{tabular}

A discrepância das estimativas entre os trabalhos citados e o executado pode ser explicada por uma série de motivos, destacando-se o número de receptoras considerado; a utilização ou não do congelamento de embriões; a estimativa do custo de aquisição, de manutenção e de venda dos animais; e, principalmente, da inserção da distribuição exponencial negativa como responsável pela geração do número de embriões viáveis por coleta. Sem esta última, sugere-se sempre a presença de uma subestimativa do custo da prenhez gerada quando índices reprodutivos similares são utilizados.

$\mathrm{Na}$ simulação, o cenário-base representa o desempenho da atividade com índices tradicionais (SPELL et al., 2001; BÓ et al., 2004; NASSER et al., 2004). Nesse caso, produziram-se 893 gestações obtendo-se custo médio de R \$1.178,19. A otimização do modelo pressupõe a análise de sensibilidade, comparando a melhor proporção de uso entre receptoras e doadoras. Tal método também foi utilizado nos traba- 
lhos de BELTRAME et al. (2007) e BARIONI et al. (2007), obtendo-se estimativas de custo inferiores aos cenários inicialmente sugeridos. No presente trabalho, a otimização da situação geraria um custo médio de R \$ 980,03 e produção de 1.681 gestações. Neste caso, um maior montante de capital e um maior número de receptoras sincronizadas por doadora em coleta deveriam ser utilizados.

Deve-se atentar que nem sempre o acréscimo na eficiência reprodutiva estabelece as menores estimativas de custo da prenhez. Este desempenho inesperado pode decorrer de problemas relacionados à ociosidade de receptoras (dado o número exagerado sendo sincronizado), à escassez de embriões, à ineficiência da técnica e, principalmente, da proporcionalidade de uso entre receptoras e doadoras.

Percebe-se que a viabilidade da TE é dependente da eficiência do protocolo e consequentemente do número de receptoras que devem ser mantidas dentro da propriedade. Dessa forma, como proposto por diversos autores (BARUSELLI et al., 2001; BÓ et al., 2001), a utilização de protocolos de sincronização por tempo fixo pode alterar a taxa de sincronização de receptoras e a composição de custos, na medida em que minimizariam o número de receptoras mantidas nas propriedades a cada coleta.

\section{CONCLUSÕES}

O cenário proposto para TE é inviável economicamente. A utilização do número de receptoras sugerido pela otimização do cenário viabiliza economicamente a técnica. Maiores estudos são necessários para avaliar o efeito do número de receptoras na viabilidade econômica da TE.

\section{REFERÊNCIAS}

ABREU FILHO, J. C. F.; SOUZA, C. P.; GONÇALVES, D. A.; CURY, M. V. Q. Finanças corporativas. 3. ed. rev. Rio de Janeiro: Editora FGV, 2003.

ANUALPEC 2005. Anuário da pecuária brasileira. São Paulo: FNP Consultoria e Comércio/Argos, 2005.

BARUSELLI, P. S.; MARQUES, M. O.; MADUREIRA, E. H.; COSTANETO, W. P.; GRANDINETTI, R. R; BÓ, G. A. Increased pregnancy rates in embryo recipients treated with CIDR-B devices and eCG. Theriogenology, v. 55, p. 157, 2001.
BARIONI, L. G.; BELTRAME, R. T.; QUIRINO, C. R.; FERNANDES, D. R. Modelos determinista e estocástico em programas de transferência de embriões em bovinos. Archivos Latinoamericanos de Producción Animal, v. 15, p. 107-113, 2007.

BELTRAME, R. T. Simulação bioeconômica da transferência de embriões em bovinos da raça Nelore. 2006.86 f. Dissertação (Mestrado) - Universidade Estadual do Norte Fluminense, Campos dos Goytacazes, 2006.

BELTRAME, R. T.; BARIONI, L. G.; MAESTRI, B. D.; QUIRINO, C. R. Economic optimization of the number of recipientes in bovine embryo transfer programs. Scientia Agricola, v. 64, p. 221-226, 2007.

BÓ, G. A.; TRÍBULO, H.; CACCIA, M.; TRÍBULO, R. Pregnancy rates in embryo recipients treated with progesterone vaginal devices and transferred without estrus detection. Theriogenology, v. 55, p. $357,2001$.

BÓ, G. A.; MORENO, L.; CUTAIA, L.; BARUSELLI, P. S.; REIS, E. L. Manipulação do ciclo estral em doadoras e receptoras de embrião bovino. Acta Scientiae Veterinariae, v. 32, p. 1-22, 2004.

FERNANDES, C. A. C. Inovulações não cirúrgicas e taxa de gestação em receptoras de embrião. Arquivo Brasileiro de Medicina Veterinária e Zootecnia, v. 51, p. 263-266, 1999.

FERNANDES C.A. C.; OBA, E.; VIANA, J. H. M; FIGUEIREDO, A. C. S.; OLIVEIRA, E. R.; VASCONCELOS, T. D.; GIOSSO, M. M. Avaliação econômica da bipartição de embriões em bovinos. Acta Scientiae Veterinariae, v. 34, p. 507, 2006.

FRIGGENS, N. C.; CHAGUNDA, M. G. G. Prediction of the reproductive status of cattle on the basis of milk progesterone measures: model description. Theriogenology, v. 64, p. 155-190, 2005 .

GALLI, C.; DUCHI, R.; CROTTI, G.; TURINI, P. bovine embryo technologies. Theriogenology, v. 59, p. 599-616, 2003.

NASSER, L. F.; REIS, E. L.; OLIVEIRA, M. A.; BÓ, G. A.; BARUSELLI P. S. Comparison of four synchronization protocols for fixed-time bovine embryo transfer in Bos indicus - Bos taurus recipients. Theriogenology, v. 62, p. 1577-1584, 2004.

OSTERGAARD, S.; FRIGGENS, N. C.; CHAGUNDA, M. G. G. Technical and economic effects of an inline progesterone indicator in a dairy herd estimated by stochastic simulation. Theriogenology, v. 64, p. 819-843, 2005.

PENEDO, R. C. A taxa interna de retorno na análise de investimentos. 1. ed. Brasília: Lettera, 2005.

PERIN, F. C. Introdução à simulação de sistemas. Campinas, SP: Editora da Unicamp, 1995. 163 p. 
REICHENBACH, H. D.; OLIVEIRA, M. A. L.; LIMA, P. F.; SANTOS, A. S.; ANDRADE, J. C. O. Transferência e criopreservação de embriões de bovinos. In: GONÇALVES, P. B. D.; FIGUEIREDO, J. R.; FREITAS, U. J. F. Biotécnicas aplicadas à reprodução animal. São Paulo: Varela, 2002. p. 127-178.

SLENNINGL, B. D.; WHEELERA, M. B. Risk evaluation for bovine embryo transfer services using computer simulation and economic decision theory. Theriogenology, v. 31, p. 653-673, 1989.
SPELL, A. R.; BEAL, W. E.; CORHA, L. R.; LAMB, C. G. Evaluete recipient and embryos factors that affect pregnancy rates of embryo transfer in beef cattle. Theriogenology, v. 56, p. 287-297, 2001.

TEDESCHI, L. O.; FOX, D. G.; SAINZ, R. D.; MEDEIROS, S. R.; BOIN, C.; BARIONI, L. G. Mathematical models in ruminant nutrition. Scientia Agricola, v. 62, n. 1, p. 76-91, 2005.

Protocolado em: 26 set. 2008. Aceito em: 25 nov. 2009. 